\title{
Synthesis and Characterization of Ruthenacarborane
}

\section{Complexes Incorporating Chelating $N$-Donor Ligands:}

\section{Unexpected Luminescence from the Complex [3-CO-}

\author{
$3,3-\kappa^{2}-\mathrm{Me}_{2} \mathrm{~N}\left(\mathrm{CH}_{2}\right)_{2} \mathrm{NMe}_{2}$-closo-3,1,2- $\left.\mathrm{RuC}_{2} \mathrm{~B}_{9} \mathrm{H}_{11}\right]$
}

Paul A. Jelliss*, Justin Mason, Jamie M. Nazzoli, Justin H. Orlando, and Albert Vinson

Department of Chemistry, Saint Louis University, St. Louis, MO 63103, USA

Nigam P. Rath

Department of Chemistry and Biochemistry, University of Missouri - St. Louis, St. Louis, MO 63128

Michael J. Shaw

Department of Chemistry, Box 1652, Southern Illinois University Edwardsville, Edwardsville, IL 62026

\section{Supporting Information}




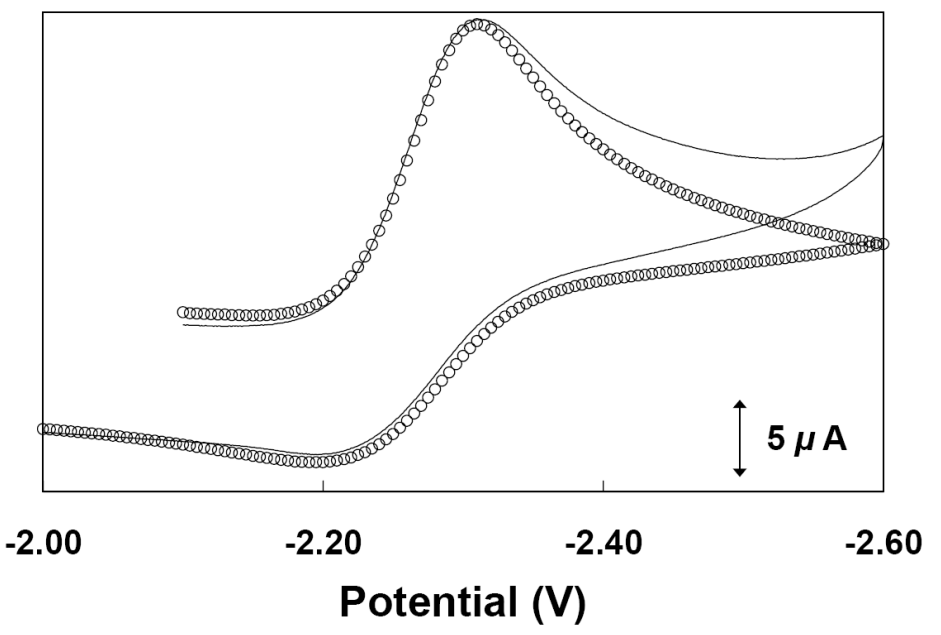

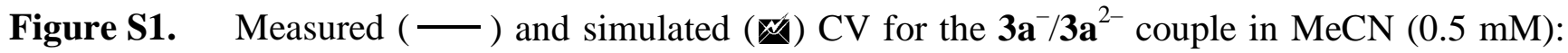
$E_{1 / 2}=-2.28 \mathrm{~V}\left(\alpha=0.50, k_{\mathrm{ET}}=0.02 \mathrm{~cm} \mathrm{~s}^{-1}\right) ;$ scan rate $=0.10 \mathrm{~V} \mathrm{~s}^{-1} ; K_{\mathrm{eq}}=10 ; k_{\mathrm{f}}=100 ; D_{3 \mathrm{a}^{-}}=1.3 \times$ $10^{-5} \mathrm{~cm}^{2} \mathrm{~s}^{-1} ; D_{3 \mathrm{a}^{2-}}=2.4 \times 10^{-6} \mathrm{~cm}^{2} \mathrm{~s}^{-1} ;$ resistance $=0 \Omega$, capacitance $=5 \mu \mathrm{F}$.

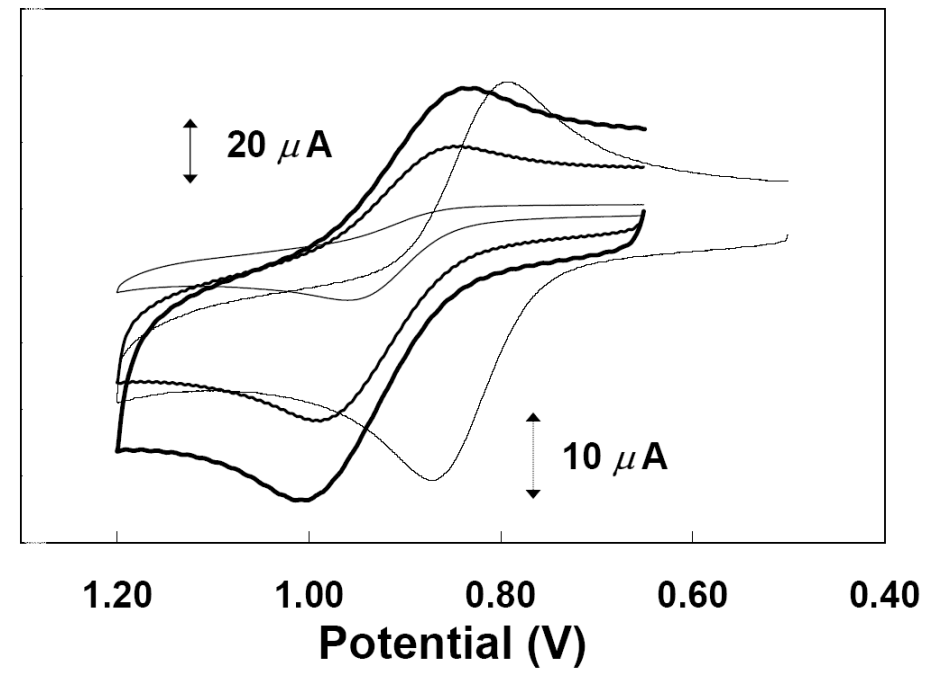

Figure S2. Oxidative-anodic $\mathrm{CVs}$ of a $\mathrm{CH}_{2} \mathrm{Cl}_{2}(1.0 \mathrm{mM})$ solution of complex 3a (toward left side, $20 \mu \mathrm{A}$ scale): $0.05 \mathrm{~V} \mathrm{~s}^{-1}(-)$ ); $0.10 \mathrm{~V} \mathrm{~s}^{-1}(-) ; 1.00 \mathrm{~V} \mathrm{~s}^{-1}$ ( - ). For comparative purposes the $\mathrm{CV}$ for complex 4 in $\mathrm{CH}_{2} \mathrm{Cl}_{2}(0.1 \mathrm{mM})$ at scan rate $0.05 \mathrm{~V} \mathrm{~s}^{-1}$ is shown toward the right side (10 $\mu$ A scale). 


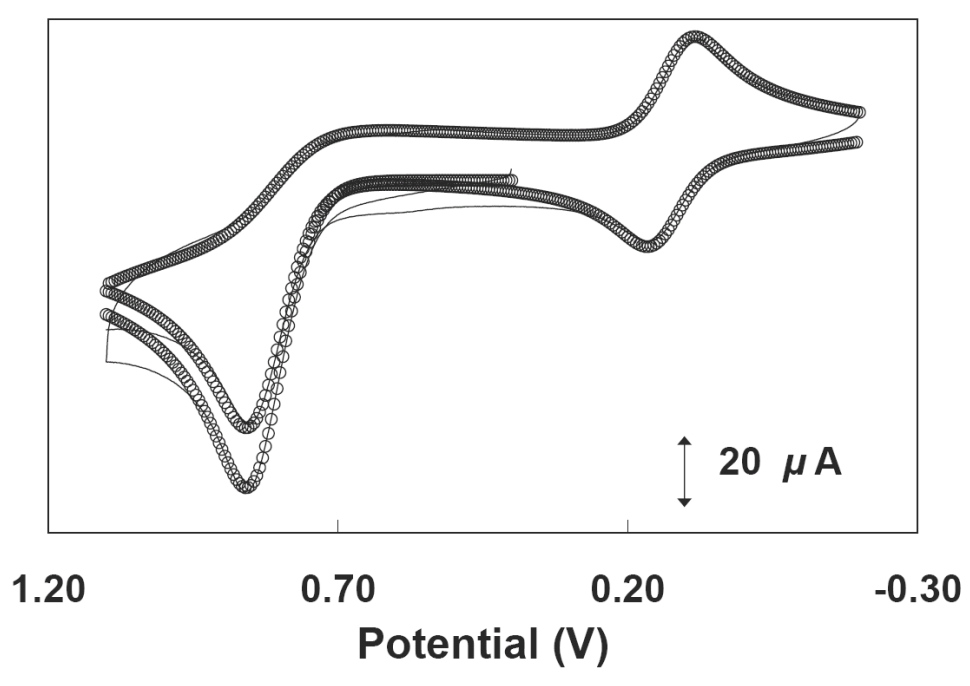

Figure S3. Measured ( - ) and simulated ( $\mathrm{CV}$ for the $\mathbf{3 c} / \mathbf{3} \mathbf{c}^{\prime^{+}} / \mathbf{3} \mathbf{c}^{\prime}$ system in $\mathrm{MeCN}(1.0 \mathrm{mM})$ : $E_{1 / 2}=0.81 \mathrm{~V}\left(\alpha=0.45, k_{\mathrm{ET}}=0.02 \mathrm{~cm} \mathrm{~s}^{-1}\right) ; E_{1 / 2}=0.11 \mathrm{~V}\left(\alpha=0.50, k_{\mathrm{ET}}=0.03 \mathrm{~cm} \mathrm{~s}^{-1}\right) ;$ scan rate $=$ $1.00 \mathrm{~V} \mathrm{~s}^{-1} ; K_{\mathrm{eq}}=50 ; k_{\mathrm{f}}=250 ; D_{3 \mathrm{c}}=2.2 \times 10^{-5} \mathrm{~cm}^{2} \mathrm{~s}^{-1} ; D_{3 \mathrm{c}^{+}}=8.0 \times 10^{-6} \mathrm{~cm}^{2} \mathrm{~s}^{-1}$ (estimated); $D_{3 \mathrm{c}^{\mathbf{c}^{+}}}=$ $8.3 \times 10^{-6} \mathrm{~cm}^{2} \mathrm{~s}^{-1} ; D_{3 \mathrm{c}^{\prime}}=2.0 \times 10^{-5} \mathrm{~cm}^{2} \mathrm{~s}^{-1}$ (estimated); resistance $=0 \Omega$, capacitance $=4 \mu \mathrm{F}$.

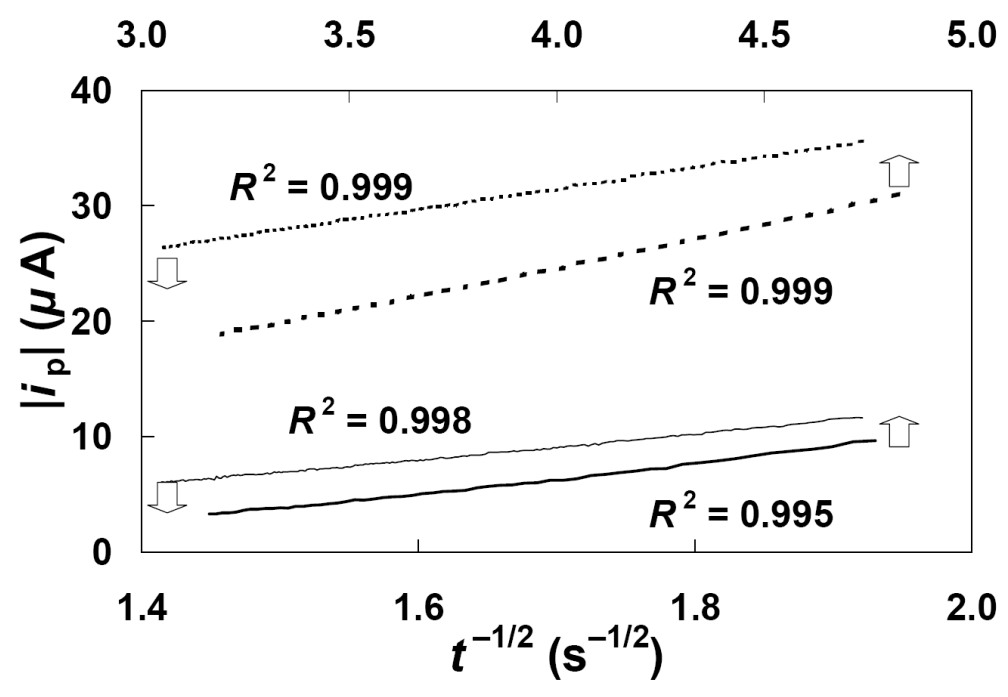

Figure S4. Cottrell plots from chronoamperometric data for complexes $\mathbf{3 c}(----), \mathbf{3 c}^{\prime^{+}}(-)$) (both lower abscissa), $\mathbf{4}(\bullet \cdot)$, and $\mathbf{4}^{+}(\boldsymbol{C})$ (both upper abscissa). 


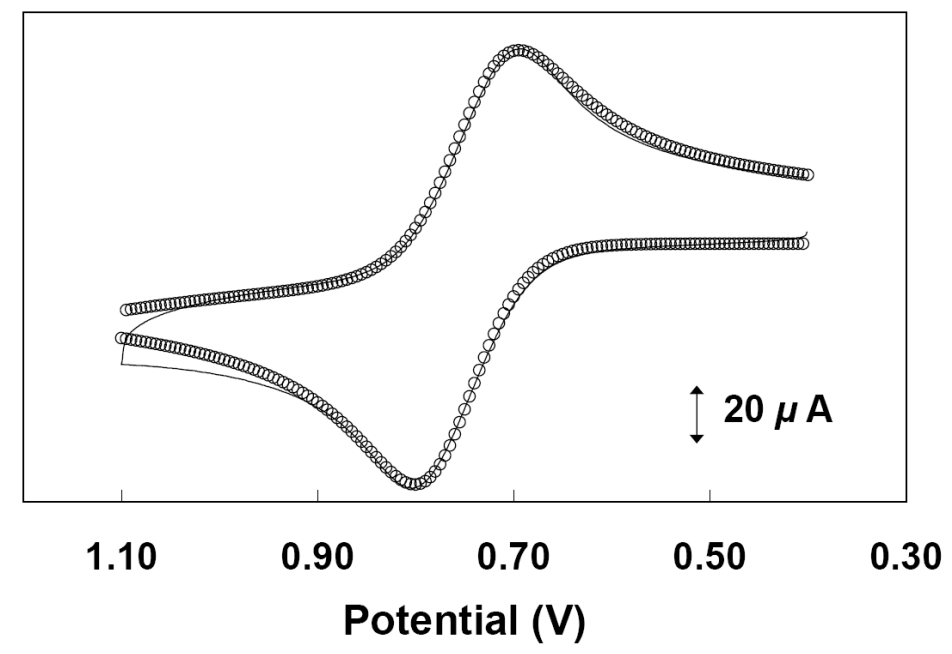

Figure S5. Measured ( - ) and simulated ( $) \mathrm{CV}$ for the $\mathbf{4}^{+} / \mathbf{4}$ couple in $\mathrm{MeCN}(1.0 \mathrm{mM}): E_{1 / 2}=$ $0.75 \mathrm{~V}\left(\alpha=0.50, k_{\mathrm{ET}}=0.03 \mathrm{~cm} \mathrm{~s}^{-1}\right) ;$ scan rate $=0.10 \mathrm{~V} \mathrm{~s}^{-1} ; K_{\mathrm{eq}}=0.4 ; k_{\mathrm{f}}=100 ; D_{\mathbf{4}}=4.4 \times 10^{-5} \mathrm{~cm}^{2}$ $\mathrm{s}^{-1} ; D_{4^{+}}=1.1 \times 10^{-5} \mathrm{~cm}^{2} \mathrm{~s}^{-1} ;$ resistance $=0 \Omega$, capacitance $=5 \mu \mathrm{F}$.

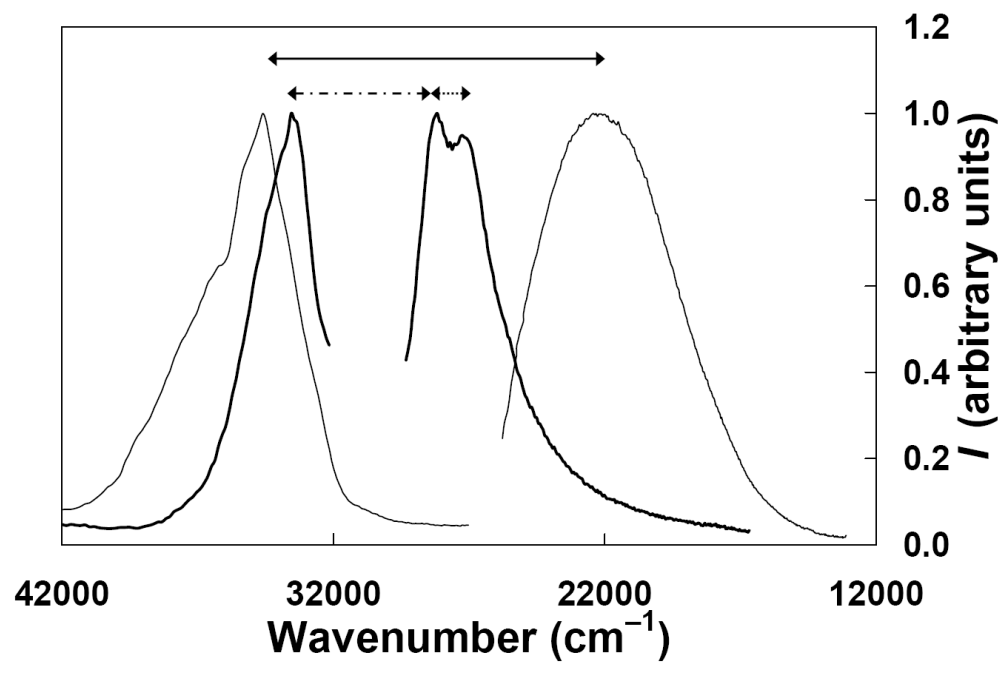

Figure S6. Photoexcitation and emission spectra measured in THF $(0.5 \mathrm{mM})$ at $298 \mathrm{~K}(\boldsymbol{C})$ and in MeTHF $(50 \mu \mathrm{M})$ at $77 \mathrm{~K}(\longrightarrow)$ plotted on an energy scale: Stokes shift $12600 \mathrm{~cm}^{-1}(\longleftrightarrow)$;

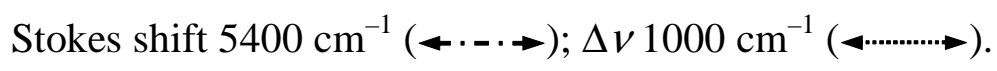

